
ОБУЧАЮЩЕГО КУРСА ПО МАТЕМАТИЧЕСКОМУ АНАЛИЗУ В СИСТЕМЕ МООDLЕ

\title{
EXPERIENCE IN DESIGNING AN ELECTRONIC TRAINING COURSE ON MATHEMATICAL ANALYSIS IN THE MOODLE SYSTEM
}

\begin{abstract}
Аннотация. В настоящее время на первый план в образовании выходит применение технологий электронного обучения (e-learning). Наиболее актуальным это является в условиях вузовского обучения, где наблюдается процесс преобладания современных педагогических технологий, в том числе технологий электронного обучения, над традиционными. В отличие от представления знаний в готовом виде при традиционном обучении, технологии электронного обучения предусматривают повышение уровня самостоятельной работы обучающихся в индивидуальном темпе, с одной стороны, предоставляя возможности для широкого общения с другими обучающимися и совместного планирования своей деятельности - с другой. Автором рассмотрены возможности использования элементов системы Moodle при освоении математической дисциплины и показано, что Moodle логично дополняет традиционную форму обучения. LMS Moodle - одна из наиболее известных и распространенных систем управления электронным обучением. Систему отличают простота и удобство использования, широкие возможности по организации образовательного процесса и контроля знаний обучающихся. В статье подробно раскрыты этапы проектирования электронного обучающего курса по дисциплине. Рассмотрены возможности системы Moodle при создании электронного курса дисциплины. Описаны методические особенности разработки информационного контента электронного обучающего курса. Обосновано, что в ходе обучения, организованного таким образом, студенты вовлечены в активную познавательную деятельность, при этом они осваивают новый вид деятельности в условиях открытого доступа к информации, знакомятся с новыми технологиями.
\end{abstract}

Ключевые слова: электронная среда; система Moodle; электронное обучение; электронный обучающий курс; обучение с веб-поддержкой; математический анализ; активные методы обучения.

Сведения об авторе: Белько Елена Станиславовна, ORCID: 0000-0002-1610-4993, канд. пед. наук, Сибирский федеральный университет, г. Красноярск, Россия, ebelko@sfu-kras.ru

About the author: Belko Elena Stanislavovna, ORCID: 0000-0002-1610-4993, Ph.D., Siberian Federal University, Krasnoyarsk, Russia, ebelko@sfu-kras.ru

Повсеместное внедрение в образовательный процесс информационно-коммуникационных технологий является общемировой тенденцией и, вполне возможно, представляет собой один из
Abstract. Currently, the use of e-learning technologies is coming to the fore in education. This is most relevant in the context of higher education, where there is a predominance of modern pedagogical technologies, including e-learning technologies, over traditional ones. In contrast to the presentation of knowledge in a ready-made form in traditional training, e-learning technologies provide for increasing the level of independent work of students at an individual pace on the one hand, providing opportunities for broad communication with other students and joint planning of their activities on the other. The author considers the possibilities of using elements of the Moodle system in the development of a mathematical discipline and shows that Moodle logically complements the traditional form of education. LMS Moodle is one of the most well-known and widespread e-learning management systems. The system is characterized by simplicity and ease of use, wide opportunities for organizing the educational process and monitoring students' knowledge. The article describes in detail the stages of designing an electronic training course for the discipline. The possibilities of the Moodle system for creating an electronic course of the discipline are considered. The article describes the methodological features of developing the information content of the e-learning course. It is reasonable that in the course of training organized in this way, students are involved in active cognitive activity, while students learn a new type of activity in an open access to information, get acquainted with new technologies.

Keywords: electronic environment; Moodle system; e-learning; e-learning course; web-based learning; mathematical analysis; active learning methods. 
самых значимых процессов, произошедших в системе образования за последние десятилетия, а также новое направление в образовании.

Российские вузы пересматривают стратегии своего развития, исходя из необходимости разрешения основных противоречий между все возрастающими потребностями динамично развивающегося информационного общества и невозможностью их удовлетворения в рамках традиционной системы образования [8].

В конце 2012 г. был принят, а с сентября 2013 г. введен в действие Федеральный закон «Об образовании в Российской Федерации», в котором говорится, что «организации, осуществляющие образовательную деятельность, вправе применять электронное обучение, дистанционные образовательные технологии при реализации образовательных программ» [9]. Сегодня ведущие университеты при организации образовательной деятельности широко применяют технологию электронного обучения [4].

Электронное обучение (e-learning) - это обучение с применением информационно-коммуникационных технологий и электронных обучающих ресурсов. Оно основано на принципе «docendo discimus» - «учусь лишь тогда, когда сам учу». Данный принцип стимулирует развитие способностей обучающихся к самостоятельному обучению и повышает их интерес к изучаемым предметам. Системы электронного обучения, или e-learning системы, часто называются системами управления обучением (Learning Management Systems - LMS). Одной из наиболее известных и распространенных систем управления электронным обучением является LMS Moodle (модульная объектно ориентированная динамическая учебная среда) [7].

Moodle - европейская система дистанционного обучения (Learning Management System LMS), которая реализует философию «педагогики социального конструктивизма» и направлена на организацию взаимодействия преподавателей и обучающихся через дистанционные курсы, осуществляющегося посредством Интернета. Основоположник, лидер и идеолог проекта - австралиец Мартин Доуджиамас (Martin Dougiamas). Систему отличают простота и удобство использования, широкие возможности по организации образовательного процесса и контроля знаний обучающихся [2].

Преподавателями института космических и информационных технологий (ИКИТ) Сибирского федерального университета (СФУ) ведется работа по проектированию и внедрению в учебный процесс электронных обучающих курсов (ЭОК) всех дисциплин. Электронные образовательные ресурсы СФУ размещаются в общеуниверситетской информационно-обучающей системе, функционирующей на базе платформы Moodle [5].

Организация образовательного процесса в электронной среде Moodle включает в себя три этапа (рис. 1) и позволяет создать единое информационное образовательное пространство для обучающихся и преподавателей в рамках электронного курса дисциплины [1].

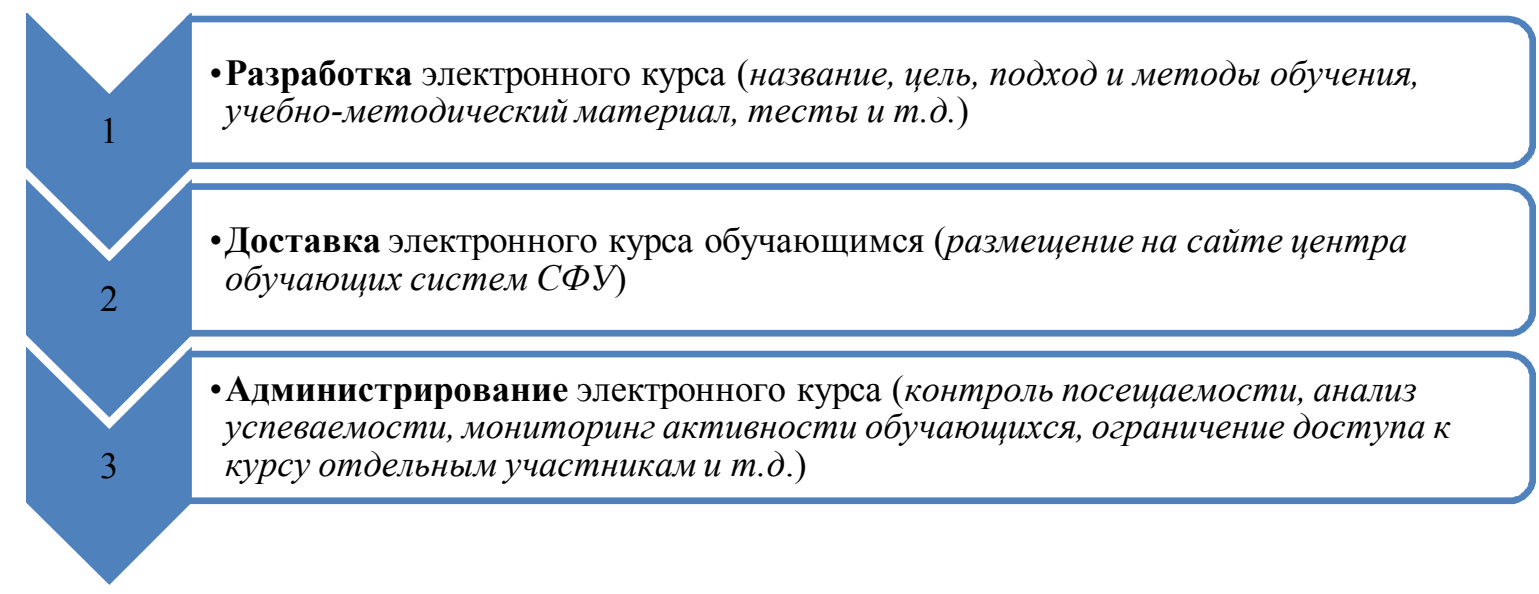

Рис. 1. Этапы организации образовательного процесса в электронной среде Моодle

С целью увеличения количества образовательных программ с применением системы электронного обучения Moodle, повышения эффективности и качества учебного процесса на основе использования различных форм его организации автором статьи был разработан и реализован 
ЭОК дисциплины «Математический анализ» для студентов бакалавров ИКИТ по направлению «программная инженерия».

Разработанный ЭОК «Математический анализ» представляет собой учебно-методический комплекс дисциплины (УМКД) и содержит: рабочую программу дисциплины; фонд оценочных средств; комплект учебно-методических материалов, обеспечивающий осуществление всех видов учебной работы, предусмотренных рабочей программой дисциплины (методические рекомендаичи по работе с электронным курсом, примерный перечень вопросов к зачету, экзамену по учебной дисииплине, электронный терминологический словарь, список основной и дополнительной литературы, информационный контент дисциплины); дополнительный материал (гиперссылки на внешние ресурсы, видеоматериаль и т. д.).

При размещении УМКД дисциплины в информационной обучающей системе СФУ создается титульный экран. Титульный экран включает в себя следующую информацию:

- личные данные об авторе и преподавателях электронного курса;

- название института;

- шифр и название образовательной программы;

- год набора и направление.

Важно отметить, что информационно-технологическая конструкция ЭОК соответствует требованию Положения об электронных образовательных ресурсах СФУ [6]. Остановимся более подробно на описании методических особенностей проектирования и реализации электронного курса «Математический анализ».

Основная задача высшего образования заключается в формировании высококвалифицированных кадров инженерных специальностей, способных к саморазвитию, самообразованию, инновационной деятельности. Решение этой задачи вряд ли возможно только путем передачи знаний в готовом виде от преподавателя к студенту. Необходимо перевести студента из пассивного потребителя знаний в активного их творца, умеющего самостоятельно добывать информацию и активно включаться в творческую исследовательскую деятельность. В свое время А. Дистервег отмечал: «Не в количестве знаний заключается образование, а в полном понимании и искусном применении всего того, что знаем» [2].

Полагаем, что внедрение личностно ориентированного и компетентностного подходов в процесс обучения способно вовлечь обучающегося в учебную деятельность по освоению рассматриваемой дисциплины. Для эффективной реализации задуманного необходимы адекватные формы учебных занятий, содержание учебной деятельности обучающихся и методы обучения.

Проектирование электронного курса начинается с разработки его структуры, которую автор выбирает самостоятельно, наполняя содержимое информационным контентом, который четко соответствует учебной программе изучаемой дисциплины. ЭОК дисциплины «Математический анализ» состоит из модулей курса, которые, в свою очередь, разбиты на темы. Каждая тема модуля ЭОК содержит лекционный (теоретический) материал по теме, задачи к лекции для самоконтроля, итоговый тест. Количество лекций совпадает с количеством недель. На каждой календарной неделе изучается соответствующая лекция. В качестве примера рассмотрена структура одного из модулей ЭОК: Модуль 5. «Интегральное исчисление функций нескольких переменных» (рис. 2).

При размещении информационного контента дисциплины система Moodle предоставляет автору возможность установить сроки их изучения (привязать к определенным датам изучения модуля, открыть только после изучения определенной темы или прохождения теста и т. д.).

Доступ обучающихся к теоретическому материалу (лекции, методические пособия, видеоматериал, презентации и т. д.) в данном электронном курсе не ограничен ни временем, ни местом. Тем самым в структуру ЭОК заложена возможность перспективно-опережающего обучения, когда студент перед аудиторной лекцией знакомится с ее содержимым в электронном курсе и имеет возможность задать уточняющие вопросы преподавателю (или обсудить их со студентами) на форуме и получить на них ответы [5]. На каждой календарной неделе изучается одна лекция.

Специфика дисциплины такова, что все математические понятия тесно связаны между собой. Из одного понятия следует другое и пропуск какого-либо из них может вызвать непонимание последующего. Решать задачи к лекции необходимо последовательно. Поэтому решение задач в модуле имеет строгую навигацию. Только после успешного решения задачи к лекиии 1 студент 
получит доступ к материалу задачи к лекиии 2 и т. д. Таким образом, переход к следующему элементу курса происходит только после завершения предыдущего.

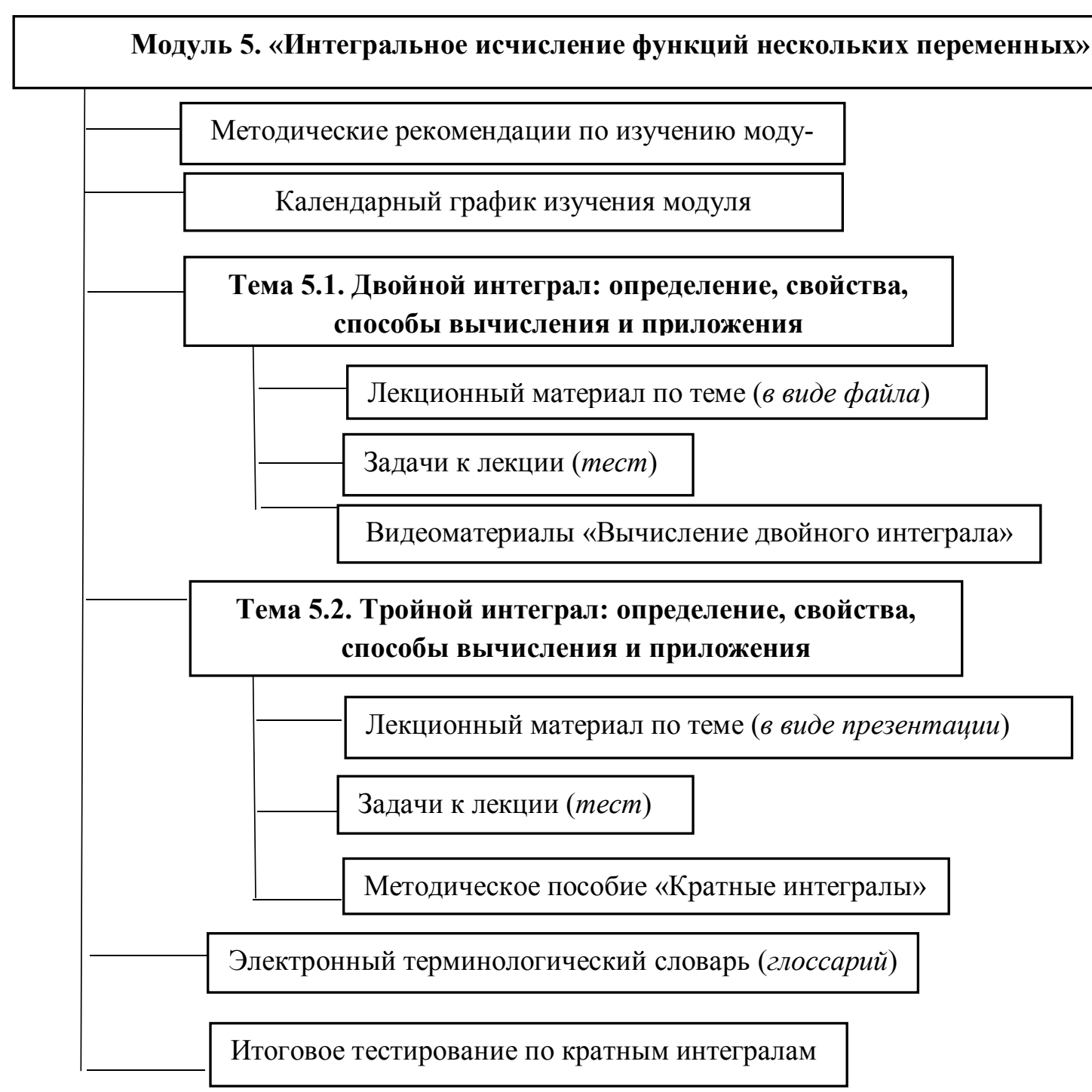

Рис. 2. Структура модуля в ЭОК «Математический анализ»

Теоретический материал в ЭОК представлен в виде лекции-презентации, видеолекиии, лекuии-конспекта (pdf-paйл), а некоторые лекции представлены в системе Moodle как элемент курса «Лекuия», представляющий собой набор страниц, где каждая страница заканчивается вопросом, на который обучающийся должен ответить (возможно чередование странии с теоретическим материалом и странии с вопросом). В случае верного ответа на вопрос обучающийся переходит на следующую страницу, в случае неверного - возвращается на предыдущую. Преподаватель, при необходимости, может комментировать неправильные ответы. Это позволяет определять маршрут обучения и не пропускать дальше без качественно усвоенного материала.

Данная форма учебного занятия относится к активным методам обучения и выполняет не только образовательную функцию, но и контрольную - обучающийся осуществляет самопроверку, а преподаватель оценивает его уровень владения теоретическим материалом, анализирует возникшие затруднения [5].

Важнейшим видом учебной деятельности, в процессе которой усваивается система математических знаний, умений и навыков, является решение задач. Роль задач в обучении математике невозможно переоценить. Они развивают логическое мышление, память, внимание, а также способствуют развитию умения кратко, правильно и четко излагать свои мысли. 
Серьезное внимание при проектировании ЭОК было уделено подбору прикладных и профессиональных задач. Необычная формулировка задач, связь с жизнью и межпредметные связи вызывают интерес у обучающихся. Их увлекает процесс поиска путей решения таких задач, что приводит к более прочному усвоению информации, так как возникают ассоциации с конкретными действиями и событиями.

Каждая учебная лекция электронного курса снабжена задачами к ней в тестовой форме, которые студент самостоятельно решает дома в системе Moodle еженедельно. Тест включает не более 10 вопросов, в зависимости от емкости лекции. Проверка правильности решения осуществляется в онлайн-режиме, обучающийся видит полученный результат и допущенные ошибки. Количество повторных попыток прохождения теста не ограничено и зависит от степени мотивированности самого обучающегося.

У обучающихся по ряду причин (низкий уровень математической подготовки, различие объема изучаемой информации в школе и вузе, высокая степень абстрактности большинства математических понятий и т. д.) разный уровень восприятия учебного материала. Некоторые обучающиеся достаточно быстро осваивают новый материал и в состоянии решать сложные задачи, а другим необходим более длительный промежуток времени. Таким студентам требуется сначала освоить решение базовых задач, чтобы процесс перехода к сложным задачам не привел к потере интереса к учебе. Одно из достоинств системы Moodle заключается в том, что она позволяет преподавателю организовать индивидуальное сопровождение студента. В удобном месте и спокойной обстановке, в удобное время и привычном для себя темпе обучающийся имеет возможность работать с информационным контентом ЭОК до тех пор, пока это необходимо ему самому. Таким образом, система Moodle - отличный тренажер для успешного освоения дисциплины, и это еще одно ее достоинство.

Промежуточный контроль по каждому модулю осуществляется в форме тестирования с помощью элемента курса «Тест» и с проверкой в онлайн-режиме. Безусловным достоинством системы является то, что Moodle выступает мощным инструментарием для создания тестов одновременно с хорошим анализатором качества теста и его составляющих - тестовых вопросов [3].

Выполнение итогового теста как обязательное условие успешного изучения модуля позволяет проверить уровень полученных знаний с помощью электронного обучающего курса по дисциплине. Максимальное количество попыток при выполнении итогового теста равно двум. При этом студент может не использовать вторую попытку, если он получил положительную оценку. В случае использования обеих попыток всегда засчитывается средняя оценка. Итоговый тест ограничен по времени и выполняется в строго установленные сроки. С помощью встроенного календаря в электронном курсе выставлены даты (начало и окончание) изучения каждого модуля дисциплины, после чего обучающимся доступен только его теоретический материал.

За решение задач к лекциям и выполнение итогового теста по модулю студенты набирают баллы, которые в сумме формируют оценку за работу в семестре. Критерии оценивания всех видов работ, выполняемых в ЭОК, были известны и доступны для обучающихся, что позволило избежать разногласий между студентом и преподавателем по вопросам полученных баллов.

ЭОК организован таким образом, что обучающиеся не имели возможности приступить к изучению следующего модуля, не получив определенное количество баллов за текущий, что стимулировало своевременное выполнение заданий и получение максимального количества баллов.

ЭОК состоит из двух частей и сопровождает изучение дисциплины «Математический анализ» в течение первого года обучения. Итоговой аттестацией по дисциплине в первом семестре является зачет, во втором - экзамен.

Система Moodle обладает еще рядом полезных возможностей, которые преподаватель курса может использовать при организации учебного процесса в электронной среде.

- Общение в ЭОК. Используя элементы курса «Пояснение» и «Форум», преподаватель может в любое время разместить объявление. Вся информация хранится в ЭОК, а значит, никуда не теряется и доступна всегда. Участники ЭОК могут обменяться сообщениями друг стругом, обсудить предложенную тему (лекиию, задачу и т. д.).

- Контроль посещаемости. У преподавателя есть возможность отслеживать посещаемость и активность студента в электронном курсе: когда заходил и в течение какого времени работал, с 
каким материалом и т.д. Также преподаватель может вовремя обратить внимание на «отстающих» студентов и принять меры.

- Контроль успеваемости. Позволяет отслеживать достижения обучающихся (баллы за все виды работ в ЭОК) с помощью «Электронного журнала». Отчет успеваемости представлен в виде таблицы, в которой баллы можно редактировать: уменьшить или увеличить в зависимости от работы студента в электронном курсе. Как показала практика, регулярная работа в ЭОК с учебным материалом положительно сказывается на успеваемости по дисциплине.

- Размешение всего информационного контента в одном месте, что вряд ли возможно при традиционном обучении. Это позволяет преподавателю использовать ЭОК для организации самостоятельной работы: изучение теоретического материала, решение задач по темам лекций, прохождение итоговых тестов по модулю. Студент, пропустивший занятия, получает возможность самостоятельно изучить материал и «нагнать» группу, так как не имеет препятствий для доступа к информационному контенту, вызванных временем или местом.

- Корректировка всего информационного контента в ЭОК возможна на протяжении всего процесса изучения дисциплины. Преподаватель может разместить дополнительные методические пособия, демонстрационные варианты аудиторных контрольных работ, видеоматериалы, презентации и т. д.

Разработанный ЭОК «Математический анализ» внедрен в учебный процесс в рамках модели электронного обучения, называемой обучением с веб-поддержкой [8].

Электронная среда Moodle используется в качестве дополнения к традиционному процессу обучения и доставки обучающимся информационного контента, организации самостоятельной работы и контроля знаний. Электронный курс является образовательным элементом, который, несомненно, обогащает традиционное обучение.

Мы полагаем, что в системе Moodle создана образовательная среда, позволяющая в корне изменить традиционные формы обучения и дающая большие возможности для повышения качества образования. С развитием Интернета и технологий электронное обучение будет все больше проникать в нашу жизнь. Электронное обучение - актуальное решение в современном стремительном экономическом мире.

\section{ЛИТЕРАТУРА}

1. Белозубов А. В., Николаев Д. Г. Система дистанционного обучения в Moodle. СПб., 2007.

2. Белько Е. С., Зыкова Т. В., Кузнецова И. В., Кытманов А. А., Тихонов С. А. Использование электронных обучающих курсов при организации самостоятельной работы студентов // Ярославский педагогический вестник. 2016. № 1. С. 107-112.

3. Белько Е. С., Зыкова Т. В., Кытманов А. А., Тихонов С. А. Синергетический эффект использования тестирования в системе Moodle при обучении математическому анализу // XV Колмогоровские чтения: Сб. статей участников Международной научно-практической конференции, посвященной памяти профессора М.И. Зайкина (г. Арзамас, 10-13 сентября 2019 г.). Арзамас, 2019. С. 46-54.

4. Зыкова Т. В., Шершнева В. А., Кацунова А. С., Вайнштейн Ю. В., Белько Е. С. Вебинары как эффективное средство обучения математике студентов вуза // Вестник Красноярского государственного педагогического университета им. В. П. Астафьева. 2017. № 2(40). С. $62-65$.

5. Кочеткова Т. О., Шершнева В. А., Зыкова Т. В., Космидис И. Ф., Сидорова Т. В., Сафонов К. В. Методические особенности проектирования и реализации электронного обучающего курса по математическому анализу // Вестник Красноярского государственного педагогического университета им. В. П. Астафьева. 2015. № 1(31). С. 49-53.

6. Положение об электронных образовательных ресурсах ФГАОУ ВПО «Сибирский федеральный университет» от 17.06.2013 // Официальный сайт ФГАОУ ВПО «Сибирский федеральный университет». URL: https://clck.ru/PZvqE (дата обращения: 30.01.2020).

7. Сатунина А. Е. Электронное обучение: плюсы и минусы // Современные проблемы науки и образования. 2006. № 1. С. 89-90.

8. Соловьев М. А., Качин С. И., Велединская С. Б., Дорофеева М. Ю. Стратегии развития электронного обучения в техническом вузе // Высшее образование в России. 2014. № 6. С. 67-76.

9. Федеральный закон от 29.12.2012 № 273-Ф3 «Об образовании в Российской Федерации». https://clck.ru/PZw5H 


\section{REFERENCES}

1. Belozubov, A. V., \& Nikolaev, D. G. (2007). Sistema distantsionnogo obucheniya v Moodle. St. Petersburg. (In Russian).

2. Belko, E. S., Zykova, T. V., Kuznetsova, I. V., Kytmanov, A. A., \& Tikhonov, S. A. (2016). Use of electronic training courses in organisation of students' independent work. Yaroslavl Pedagogical Bulletin, (1), $107-112$. (In Russian).

3. Belko, E. S., Zykova, T. V., Kytmanov, A. A., \& Tikhonov, S. A. (2019). Sinergeticheskii effekt ispol'zovaniya testirovaniya $\mathrm{v}$ sisteme Moodle pri obuchenii matematicheskomu analizu. In XV Kolmogorovskie chteniya: sb. statei uchastnikov Mezhdunarodnoi nauchno-prakticheskoi konferentsii, posvyashchennoi pamyati professora M.I. Zaikina (g. Arzamas, 10-13 sentyabrya 2019 g.), Arzamas. 46-54. (In Russian).

4. Zykova, T. V., Shershneva, V. A., Katsunova, A. S., Vainshtein, Yu. V., \& Belko, E. S. (2017). Webinars as an effective tool of teaching mathematics to students in higher school. Bulletin of Krasnoyarsk State Pedagogical University, (2 (40)), 62-65. (In Russian).

5. Kochetkova, T. O., Shershneva, V. A., Zykova, T. V., Kosmidis, I. F., Sidorova, T. V., \& Safonov, K. V. (2015). Methodological aspects of the design and implementation of e-learning course on calculus. Bulletin of Krasnoyarsk State Pedagogical University, (1 (31)), 49-53. (In Russian).

6. Polozhenie ob elektronnykh obrazovatel'nykh resursakh FGAOU VPO "Sibirskii federal'nyi universitet" ot 17.06.2013 g. Ofitsial'nyi sait FGAOU VPO “Sibirskii federal'nyi universitet”. https://clck.ru/PZvqE

7. Satunina, A. E. (2006). Computer traning: advantages and disadvantages. Sovremennye problemy nauki $i$ obrazovaniya, (1), 89-90. (In Russian).

8. Solovev, M. A., Kachin, S. I., Veledinskaya, S. B., \& Dorofeeva, M. Yu. (2014). E-learning strategy at technical university. Higher education in Russia, (6), 67-76. (In Russia).

9. Federal'nyi zakon ot 29.12.2012 N273-FZ “Ob obrazovanii v Rossiiskoi Federatsii”. https://clck.ru/PZw5H

Белько Е. С. Опыт проектирования электронного обучающего курса по математическому анализу в системе Moodle // Вестник Нижневартовского государственного университета. 2020. № 3. С. 4-10.

https://doi.org/10.36906/2311-4444/20-3/01

Belko, E. S. (2020). Experience in designing an electronic training course on mathematical analysis in the Moodle system. Bulletin of Nizhnevartovsk State University, (3). 4-10. (In Russian). https://doi.org/10.36906/2311-4444/20-3/01 